\title{
Incidence and Measures to Prevent Intradialytic Hypotension in Patients on Maintenance Hemodialysis in a Tertiary Care Centre in India
}

\section{Malleshappa Pavan ${ }^{1 *}$, Ravi Ranganath ${ }^{2}$, Anup P Chaudhari ${ }^{2}$, Ashwinikumar Aiyangar $^{3}$, Keerti L Upadhayaya ${ }^{4}$ and Hemant J Mehta ${ }^{4}$}

${ }^{1}$ Consultant Nephrologist, Vaatsalya Hospital, Bharathi Healthcare Complex, R C Road, Hassan, Karnataka state, India

${ }^{2}$ Senior Registrar, Department of Nephrology, Lilavati Hospital and Research Centre, Mumbai, India

${ }^{3}$ Consultant Nephrologist, Global Hospital, Lakdi-ka-pul, Hyderabad, India

${ }^{4}$ Consultant Nephrologist, Lilavati Hospital and Research Centre, Mumbai, India

\begin{abstract}
Objective: Hypotension is quite common in any dialysis unit and despite significant improvements of hemodialysis techniques in the recent years; the frequency of recurrent intradialytic hypotension episodes has remained nearly unchanged.
\end{abstract}

Our study is aimed to define the pathogenesis of intradialytic hypotension and attempts to describe effective measures for the hypotension prone hemodialysis patients on an individual basis.

Design: This is a prospective study of 100 hemodialysis patients observed over 12 months at the Lilavati Hospital and Research Centre, Mumbai, India. Subjects with more than two episodes/month of hypotension on dialysis were diagnosed as having IDH.

Results: Intradialytic hypotension was found in $18 \%$ of subjects. Incorrectly assessed ideal body weight was found to be the commonest cause of intradialytic hypotension. Volume assessment and adjusting the ideal body weight was found to be central to the problem of preventing intradialytic hypotension.

Conclusion: This study shows that intradialytic hypotension is a common phenomenon, even in a tertiary care centre. Preventive strategies should be developed in each unit to decrease the future risk for intradialytic hypotension in specific patients.

\section{Introduction}

Hypotension is quite common in any dialysis unit and infusing saline comes as a knee-jerk reaction to fall in BP. Despite significant improvements of hemodialysis (HD) techniques in the recent years, the frequency of recurrent intradialytic hypotension (IDH) episodes has remained nearly unchanged with the incidence of 20 to $30 \%$ of treatments [1,2]. IDH is defined as a symptomatic decrease of more than $30 \mathrm{mmHg}$ in systolic blood pressure or as an absolute systolic blood pressure under $90 \mathrm{mmHg}$ [3]. IDH was defined as per K/DOQI guidelines as decrease in systolic blood pressure $>20 \mathrm{~mm} \mathrm{Hg}$ or a decrease in mean arterial pressure (MAP) by $10 \mathrm{~mm} \mathrm{Hg}$ associated with clinical events and need for nursing intervention [4]. Decades of research revealed that the cause of IDH is multifactorial. First described in 1970 [4], Bergström showed in 1978 that intravascular hypovolemia, due to a large volume of ultrafiltration in too short a period of time, was not the sole cause of IDH [5] IDH is the clinical manifestation of an imbalance between the decreases in plasma volume during dialysis and the counter regulatory cardiovascular hemodynamic and neurohumoral mechanisms [6-9]. Our study is aimed to define the pathogenesis of IDH and thereby to apply the most appropriate protective strategies for the hypotension prone hemodialysis patient on an individual basis.

\section{Materials and Methods}

- This is a prospective study conducted at the Lilavati Hospital and Research Centre, Mumbai, India from 1/1/2009 to $31 / 12 / 2009$

- 100 subjects who were on maintenance hemodialysis (4 hours/3 per week) observed over 12months.

- IDH was defined as per K/DOQI guidelines as decrease in systolic blood pressure $>20 \mathrm{~mm} \mathrm{Hg}$ or a decrease in mean arterial pressure (MAP) by $10 \mathrm{~mm} \mathrm{Hg}$ associated with clinical events and need for nursing intervention. Subjects with more than two episodes/month of hypotension on dialysis were diagnosed as having IDH.

- Vital statistics, comorbidities, estimated dry weight, pre and post dialysis weight, interdialytic weight gain and medications were recorded. Diet history including salt and water intake were recorded. Complete Blood Count and Iron studies were done in all patients. 2D-Echocardigraphy and assessment for autonomic dysfunction using valsalva maneuver was performed in all patients.

\section{Interventions applied included the following}

- Volume status assessment, salt and fluid restriction to minimize interdialytic weight gain up to $0.8-1 \mathrm{~kg}$ per day, abstaining from food on dialysis, anemia correction, Cardiac evaluation with 2DEchocardiography \& Inferior vena cava measurement, modifying schedules of antihypertensives and nitrates, ultrafiltration (UF)/sodium profiling, increasing dialysate sodium, decreasing dialysate temperature, and use of vasoconstrictors (vasopressin or midodrine) in a stepwise manner according to the etiology of IDH.

*Corresponding author: Dr. Malleshappa Pavan, Consultant Nephrologist Vaatsalya Hospital, Bharathi Healthcare Complex, R C Road, Hassan- 573201, Karnataka State, India, Tel: (91) 8172-251707; E-mail: dr_pavanm@yahoo.co.in

Received July 07, 2011; Accepted July 29, 2011; Published August 10, 2011

Citation: Pavan M, Ranganath R, Chaudhari AP, Aiyangar A, Upadhayaya KL, et al. (2011) Incidence and Measures To Prevent Intradialytic Hypotension in Patients on Maintenance Hemodialysis In a Tertiary Care Centre in India. J Nephrol Therapeutic 1:101. doi:10.4172/2161-0959.1000101

Copyright: (C) Pavan M, et al. This is an open-access article distributed under the terms of the Creative Commons Attribution License, which permits unrestricted use, distribution, and reproduction in any medium, provided the original author and source are credited. 
Citation: Pavan M, Ranganath R, Chaudhari AP, Aiyangar A, Upadhayaya KL, et al. (2011) Incidence and Measures To Prevent Intradialytic Hypotension in Patients on Maintenance Hemodialysis In a Tertiary Care Centre in India. J Nephrol Therapeutic 1:101. doi:10.4172/21610959.1000101

Page 2 of 4

\section{Results}

Hundred patients were included in this study. Their demographics are shown in (Table 1). Of the 100 patients studied, 18 patients were found to have hemodynamically significant IDH (Figure 1). Diabetic nephropathy was the leading cause $(61 \%)$ of ESRD among these patients (Figure 2). Factors responsible for the pathogenesis of IDH include: incorrect dry weight, poor cardiac function, excess weight gain, medications (antihypertensives and nitrates), consumption of food while on $\mathrm{HD}$, anemia, autonomic neuropathy and unknown causes (Table 2).

Appropriate interventions that proved effective in preventing the episodes of hypotension during hemodialysis include: increasing the ideal body weight, use of vasoconstrictors, modifying the medications (antihypertensives), limiting interdialytic weight gain, avoiding food consumption while on $\mathrm{HD}$, coronary revascularisation and correction of anemia (Figure 3).

\section{Discussion}

Intradialytic hypotension continues to be a leading problem, and its incidence varies from 20 to $30 \%$. Intradialytic hypotension not only causes discomfort, but also increases mortality. Risk factors for IDH are both age and comorbidity dependent. The population at risk includes individuals with diabetes (autonomic dysfunction); individuals with left ventricular hypertrophy (LVH) and diastolic dysfunction, a history of prior myocardial infarction, or cardiovascular intervention; individuals with symptomatic coronary heart disease; individuals with high interdialytic weight gains ( $>3 \%$ of body weight); and dialysis patients who are anephric [10]. In our study IDH occurred in $18 \%$ of

\begin{tabular}{|l|l|}
\hline Parameters & Mean values \\
\hline Age (years) & $50.5(30-70)$ \\
\hline Sex & Males- 68\%: Females- 32\% \\
\hline Time on dialysis (years) & $4(1-10)$ \\
\hline Nephropathy & $44.5 \%$ \\
Diabetic nephropathy & $7.6 \%$ \\
Hypertensive nephrosclerosis & $5.1 \%$ \\
Glomerulonephritis & $24.4 \%$ \\
Tubulointerstitial nephritis & $18.4 \%$ \\
\hline Unknown causes & $60.1 \mathrm{Kg}(50-89)$ \\
\hline Dry weight & $246 \mathrm{~min}(220-260)$ \\
\hline Duration of hemodialysis per session & $8.07 \mathrm{ml} / \mathrm{min}$ \\
\hline Ultrafiltration rate per session & $9.13 \mathrm{~g} / \mathrm{dl}(7.2-13.6)$ \\
\hline Hemoglobin & \\
\hline 2-D Echocardiography $(\mathrm{n}=16)$ & $77 \%$ \\
\hline Left ventricular hypertrophy (LVH) & $89.8 \%$ \\
\hline Poor systolic function & $78.7 \%$ \\
\hline Poor diastolic function & \\
\hline
\end{tabular}

Table 1: Demographic variables of the patients.

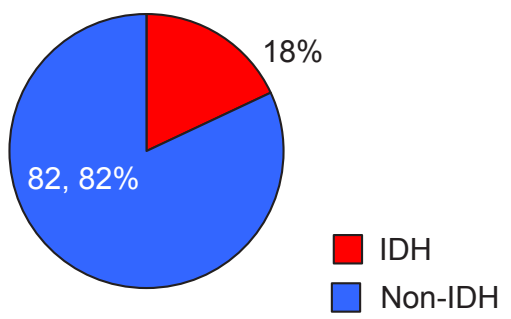

$82 \%$

Figure 1: Percentage of patients with Intradialytic hypotension (IDH).

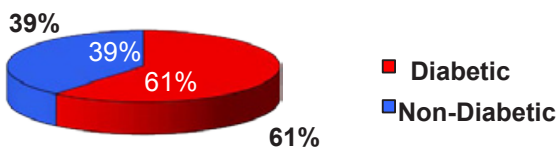

Figure 2: Percentage of diabetic and non-diabetic patients with IDH.

\begin{tabular}{|l|l|}
\hline Etiology & Percentage (\%) \\
\hline Incorrect ideal body weight (dry weight) & 33 \\
\hline Poor cardiac function & 16 \\
\hline Excess interdialytic weight gain & 11 \\
\hline Medications & 11 \\
\hline Food on HD & 11 \\
\hline Autonomic neuropathy & 6 \\
\hline Anemia & 6 \\
\hline Unknown causes & 6 \\
\hline
\end{tabular}

Table 2: Etiologies for IDH.

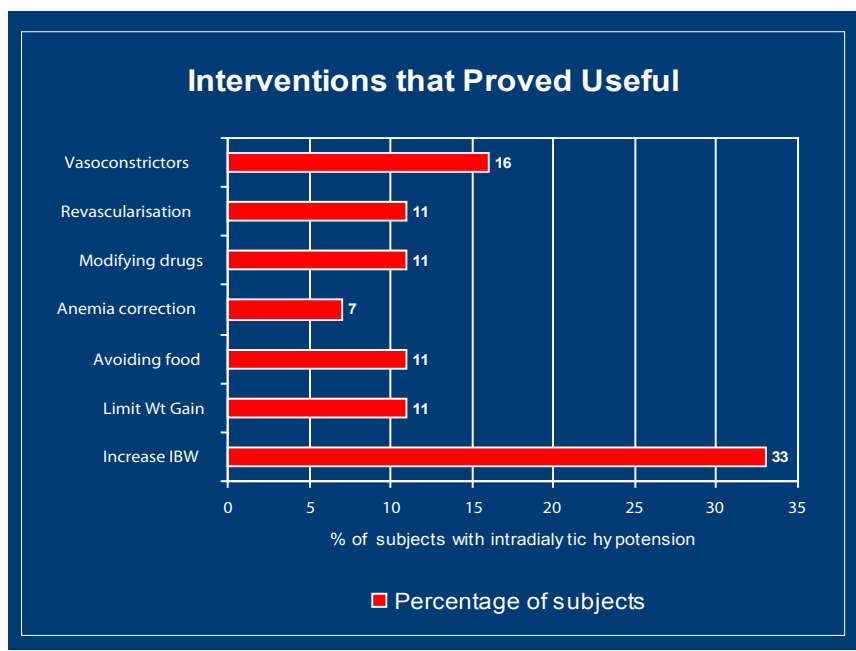

Figure 3: Interventions that proved useful in preventing IDH.

patients. Diabetic nephropathy was the leading cause of ESRD (61\%) in our patients with IDH. In our study poor cardiac function accounted for $16 \%$, interdialytic weight gain for $11 \%$ and autonomic dysfunction for $6 \%$ of patients with IDH.

In addition to age and comorbidity risk determinants for the IDH patient, volume removal requirements per treatment, although not well defined, seemingly pose a risk for IDH in a sizable number of people. A reported "safe zone" for UF per treatment is less than $3 \%$ of body weight [11]. Exceeding this 3\% safe zone for the rate of volume removal increases the risk for IDH. New dialysis patients started on HD in the United States have an approximate average safe zone UF of 1.8 to 2.3 $\mathrm{kg} /$ treatment based on mean baseline weight across all age categories of age [12] and yet the standard kilogram interdialytic weight gain pattern that typical patients present with requires UF rates higher than the accepted safe zone, thus increasing their risk for IDH. Incorrectly assessed ideal body weight followed by excess removal of UF is the commonest cause IDH (33\%) in our study.

IDH episodes may resolve with treatment in the unit or persist even after the patient leaves the unit and returns home. Theoretically, persistent hypotension after treatment completion may provide an 
Citation: Pavan M, Ranganath R, Chaudhari AP, Aiyangar A, Upadhayaya KL, et al. (2011) Incidence and Measures To Prevent Intradialytic Hypotension in Patients on Maintenance Hemodialysis In a Tertiary Care Centre in India. J Nephrol Therapeutic 1:101. doi:10.4172/21610959.1000101

Page 3 of 4

explanation for patients who sustain an insult or die sometime later at home. Patients experiencing IDH who are usually hypertensive may resume antihypertensive medications at home when the BP is still low. This could lead to further nocturnal decreases in BP that increase the risk for an ischemic event [13]. Improper dosage and frequency of antihypertensive medications administration accounted for $11 \%$ of IDH in our patients.

Detailed hemodynamic analysis of IDH suggests 2 important things. (1) There is no sudden decrease in plasma volume just before a hypotensive episode. (2) IDH seems to be due to a decrease in cardiac output engendered by reduced cardiac filling [14,15]. Most of the $3 \mathrm{~L}$ or so of plasma volume resides in the veins, and several organ perfusion systems, notably skin and splanchnic, contain veins that can markedly alter their capacity [16]. A slight loss in venous tone in either of these systems can result in a marked ebbing of flow return to the heart with loss of cardiac filling and a resultant decrease in cardiac output. Food ingestion during dialysis will increase splanchnic blood flow and may cause hypotension by causing splanchnic blood pooling [17]. Food ingestion accounted for $11 \%$ of IDH in our study. Arteriolar vasoconstriction will help limit pooling of blood in veins, thereby helping to maintain cardiac filling and cardiac output. Local tissue ischemia, via generation of adenosine or other local mediators, may aggravate hypotension, and methods that help maintain blood oxygencarrying capacity and oxygenation (eg, maintaining an adequate level of hematocrit) might maintain blood pressure by reducing tissue ischemia [6]. Anemia contributed to $6 \%$ of IDH in our patients.

Specific prevention and treatment approaches can be matched to the patient's individual physiologic events responsible for IDH $[18,19]$. The different components of the dialysis prescription can be modified to help address the various causes of IDH. Multiple pharmacologic agents are also available for use in treating IDH. These agents can be used both to avoid IDH events and potentially to decrease the frequency of acute interventions necessary once the blood pressure (BP) decreases and the patient's symptoms are apparent [20]. Educating the dialysis staff and "at-risk" patients can aid in the early recognition and timely treatment of IDH events. The number of patients at risk for IDH and prolonged hypotension has increased with the advancing mean age of dialysis patients and the multiple comorbidities with which endstage renal disease (ESRD) patients present. Considering the potential vascular risk low BP imparts, IDH can no longer be treated as a benign condition [20].

At-risk patients ${ }^{21}$ can develop specific BP patterns with either subtle or no symptoms initially. Patients should be encouraged to notify the unit personnel if they begin to feel nauseated or diaphoretic or if they experience muscle cramps, visual abnormalities, or chest pain. The sooner the unit personnel address such symptoms, the quicker the BP decrease can be rectified. The patient's standard HD should be reviewed with specific attention to the trend in percent interdialytic weight change, planned total UF, UF rate per hour, dialysate sodium concentration, dialysate calcium concentration, and dialysate temperature [20]. The use of a higher dialysate sodium concentration $(>140 \mathrm{mEq} / \mathrm{L})$ is an effective means to ensure adequate vascular refilling and has proved to be among the most efficacious and best tolerated therapies for episodic hypotension. Sodium modeling is a technique in which the dialysate sodium concentration is varied during the course of the procedure. Most common, a high dialysate sodium concentration is used initially with a progressive reduction toward isotonic or even hypotonic levels by the end of the procedure. This method of sodium control allows for a diffusive $\mathrm{Na}$ influx early in the session to prevent the rapid decline in plasma osmolality as a result of the efflux of urea and other small molecular weight solutes. During the remainder of the procedure, when the reduction in osmolality accompanying urea removal is less abrupt, the dialysate sodium level is set at a lower level. Higher dialysate sodium concentrations, whether fixed or modeled, carry the risk for sodium accumulation, leading to stimulation of thirst, increased fluid gain, and hypertension in the interdialytic period. Ultrafiltration profiling is the deliberate use of a high rate of ultrafiltration in the initial part of the treatment, when the volume of interstitial fluid available for vascular refilling is maximal, and then sequentially decreasing the rate so as to parallel the anticipated fall in interstitial fluid volume. Recent studies suggest this approach is particularly effective when combined with sodium modelling [21]. Adjusting dialysis prescription with combined sodium modelling and ultrafiltration profiling and limiting interdialytic weight gains helped in decreasing the frequency of IDH in $44 \%$ of patients in our study. A number of interventions can be relied on to decrease the overall risk of the patient for future IDH. These consist of adjusting pre-HD hypertension medications, and aggressively treating anemia to optimize blood volume [20]. These interventions proved effective in our patients as well. Avoiding antihypertensive medications on the day of dialysis was effective in $11 \%$ and correction of anemia was effective in $7 \%$ of patients in preventing recurrent IDH.

An acute BP decrease during dialysis may signal underlying coronary disease. Patients with underlying coronary disease who develop IDH, especially if the BP is not corrected to baseline, should undergo a work-up for ischemic cardiovascular disease [20]. Coronary revascularisation helped to prevent IDH in $11 \%$ of patients in our study.

Pharmacologic interventions should be considered for patients who require repeat intervention for IDH on HD. A number of medications are available (caffeine, sertraline, midodrine, vasopressin, etc) and have been safely used in ESRD patients. The most common approach to overcome impaired sympathetic activity has been to administer other vasoconstrictors to increase peripheral vascular resistance.

A systematic review of the literature [22] revealed that 2.5 to 10 mg of the selective $a-1$ adrenergic agonist midodrine was proved to be an effective treatment in some patients with frequent intradialytic hypotension. This drug may also have the additional benefit of better preserving cerebral blood flow in patients with orthostatic hypotension after hemodialysis [23]. Vasopressin was recently reported to be an effective agent to support BP during volume removal. Previous observations found that plasma vasopressin levels do not significantly increase during ultrafiltration dialysis despite an anticipated unloading of baroreceptors. In a randomized, doubleblind, placebo-controlled trial, the continuous administration of subpressor doses of vasopressin provided for greater hemodynamic stability even when target fluid loss was set to be increased by $0.5 \mathrm{~kg}$ over the baseline prescription [24]. An optimal approach may require a combination of dialysis prescription changes and oral medications [25]. We used midodrine at a dose of 10 $\mathrm{mg}$ one to two hours before the initiation of dialysis, with increasing the dose up to $40 \mathrm{mg}$. We used vasopressin infusion 0.1 to $0.3 \mathrm{mU} /$ $\mathrm{kg} / \mathrm{min}$ during hemodialysis session. Usage of these medicines helped to prevent IDH in $16 \%$ of our study subjects and helped in achieving the target increase in fluid removal. Eating during dialysis should be discouraged in patients with a risk for IDH. This approach proved effective in $11 \%$ of patients in our study. 
Citation: Pavan M, Ranganath R, Chaudhari AP, Aiyangar A, Upadhayaya KL, et al. (2011) Incidence and Measures To Prevent Intradialytic Hypotension in Patients on Maintenance Hemodialysis In a Tertiary Care Centre in India. J Nephrol Therapeutic 1:101. doi:10.4172/21610959.1000101

Page 4 of 4

\section{Conclusion}

Preventive strategies [26] should be developed in each unit to decrease the future risk for IDH in specific patients. Nursing staff and physicians can help educate the HD patient as to risk factors and the short-term and long-term implications for the patient with hypotension. A critical pathway should be implemented in individual units to help problem-solve why patients are experiencing IDH. Hypotension should be recognized and be part of the minimal data set for all patients at the start of each dialysis session (prior event record). Considering the importance of hypotension in overall patient survival, the percentage of patients in each unit who experience IDH and/or present with low BP (systolic BP $<110 \mathrm{~mm} \mathrm{Hg}$ ) should be tracked as a quality assurance initiative.

\section{References}

1. Bregman H, Daugirdas JT, Ing TS, Daugirdas JT, Blake PG, et al. (2001) Complications during hemodialysis. In: Handbook of Dialysis, $3^{\text {rd }}$ Ed.148-68.

2. Zuchell P, Santoro A (1993) Dialysis-induced hypotension: A fresh look at pathophysiology. Blood Purif 11: 85-98.

3. Kooman J, Basci A, Pizzarelli F (2007) EBPG guideline on hemodynamic instability. Nephrol Dial Transplant 2: 22-44

4. Kim KE, Neff M, Cohen B, Somerstein M, Chinitz J, et al. (1970) Blood volume changes and hypotension during hemodialysis. Trans Am Soc Artif Intern Organs 16: 508-514

5. Bergstrom J (1978) Ultrafiltration without dialysis for removal of fluid and solutes in uremia. Clin Nephrol 9: 156-164.

6. Daugirdas JT (2001) Pathophysiology of dialysis hypotension: an update. Am J Kidney Dis 38: 11-17

7. Passauer J, Bussemaker E, Gross P (1998) Dialysis hypotension: do we see the end of tunnel? Nephrol Dial Transplant 13: 3024-29.

8. Sherman RA (2002) Intradialytic hypotension: an over-view of recent, unresolved and overlooked issues. Semin Dial 15: 141-143.

9. Andrulli S, Colzani S, Mascia F, Lucchi L, Stipo L, et al. (2002) The role of blood volume reduction in the genesis of intradialytic hypotension. Am J Kidney Dis 40: $1244-1254$

10. Schreiber M (2001) Clinical dilemmas in dialysis: managing the hypotensive patient setting the stage. Am J Kidney 38: 1-10.

11. Jahn H, Brandt C, Schmitt R, Schohn D, Colotte J, et al. (1993) Hemodynamic criteria for adequacy of volume regulation and prevention of myocardial impairment in chronic hemodialysis patients. Contrib Nephrol 103:148-59.

12. US Renal Data System: USRDS 2000 Annual Data Report. The National Institutes of Health, National Institute of Diabetes and Digestive and Kidney Diseases 2000, Bethesda.

13. Amar J, Vernier I, Rossignol E, Bongard V, Arnaud C, et al. Nocturnal blood pressure and 24-hour pulse pressure are potent indicators of mortality in hemodialysis patients. Kidney Int 2000; 57: 2485-91.

14. Maeda K, Fujita Y, Shinzato T, Morita H, Kobayakawa H, et al. (1989) Mechanism of dialysis-induced hypotension. ASAIO Trans 35: 245-247.

15. Nakamura Y, Ikeda T, Takata S, Yokoi H, Hirono M, et al. (1991) The role of peripheral capacitance and resistance vessels in hypotension following hemodialysis. Am Heart J 121: 1170-1177.

16. Rothe CF (1983) Reflex control of veins and vascular capacitance. Physiol Rev 63: 1281-1342

17. Kooner JS, Raimbach S, Watson L, Bannister R, Part S, et al. (1989) Relationship between splanchnic vasodilation and postprandial hypotension in patients with primary autonomic failure. J Hypertens 7: 40-41.

18. Van der Sande FM, Kooman JP, Leunissen KM (2000) Intradialytic hypotension-New concepts on an old problem. Nephrol Dial Transplant 15: 1746-1748.
19. Zucchelli P, Santoro A (1999) The management of hypotension in dialysed patients. Miner Electrolyte Metab 25: 105-108.

20. Schreiber M (2001) Clinical Case-Based Approach to Understanding Intradialytic Hypotension. Am J Kidney Dis 38: 37-47.

21. Zhou YL, Liu HL, Duan XF, Yao Y, Sun Y, et al. (2006) Impact of sodium and ultrafiltration profiling on haemodialysis-related hypotension. Nephrol Dial Transplant 21: 3231-3237.

22. Prakash S, Garg AX, Heidenheim AP, House AA et al. (2004) Midodrine appears to be safe and effective for dialysis-induced hypotension: A systematic review. Nephrol Dial Transplant 19: 2553-2558.

23. Fujisaki K, Kanai H, Hirakata H, Nakamura S, Koga Y, et al. (2007) Midodrine hydrochloride and L-threo-3,4-dihydroxy-phenylserine preserve cerebral blood flow in hemodialysis patients with orthostatic hypotension. Ther Apher Dial 11 49-55.

24. Van der Zee S, Thompson A, Zimmerman R, Lin J, Huan Y, et al (2007) Vasopressin administration facilitates fluid removal during hemodialysis. Kidney Int 71: 318-324.

25. Alappan R, Cruz D, Abu-Alfa AK, Mahnensmith R, Perazella MA (2001) Treatment of severe intradialytic hypotension with the addition of high dialysate calcium concentration to midodrine and/or cool dialysate. Am J Kidney Dis 37: 294-299.

26. Donauer J, Ko"Iblin D, Bek M, Krause A, Bo"hler J (2000) Ultrafiltration profiling and measurement of relative blood volume as strategies to reduce hemodialysis-related side effects. Am J Kidney Dis 36: 115-123. 